\title{
NK cell lymphoma, nasal type, with massive lung involvement: a case report
}

\author{
Anamarija Morovic • Igor Aurer • Snjezana Dotlic • \\ Dennis D. Weisenburger • Marin Nola
}

Received: 12 August 2009 /Accepted: 17 November 2009/Published online: 24 March 2010

(C) Springer-Verlag 2010

\begin{abstract}
Extranodal NK/T cell lymphoma, nasal type, is an Epstein-Barr virus-associated lymphoma that most commonly involves the nasal cavity and upper respiratory tract. Lung involvement by NK/T cell lymphoma is rare and seldom reported in the literature. We describe the unusual case of a 41-year-old male with NK cell lymphoma, nasal type, who presented with massive secondary lung involvement 2.5 years after the detection of a retroperitoneal mass. The diagnosis was made by open lung biopsy. Despite aggressive treatment, the patient died shortly after the initiation of therapy. Lung involvement by $\mathrm{NK} / \mathrm{T}$ cell lymphoma occurs most commonly as part of widely disseminated disease and carries a poor prognosis for the patient. Novel agents and innovative therapies need to be developed for this aggressive lymphoma.
\end{abstract}

\section{A. Morovic $(\square)$}

Department of Pathology and Laboratory Medicine,

University of Cincinnati College of Medicine,

231 Albert Sabin Way,

Cincinnati, OH 45267, USA

e-mail: moroviaa@uc.edu

\section{Aurer}

Department of Internal Medicine,

Zagreb University Hospital Center,

Kispaticeva 12,

Zagreb 10000, Croatia

\section{D. Weisenburger}

Department of Pathology and Microbiology,

University of Nebraska Medical Center,

983135 Nebraska Medical Center,

Omaha, NE 68198, USA

S. Dotlic $\cdot$ M. Nola

Department of Pathology, Zagreb University Hospital Center,

Kispaticeva 12,

Zagreb 10000, Croatia
Keywords Extranodal NK/T lymphoma .

Lung involvement $\cdot$ Epstein-Barr virus

\section{Introduction}

Extranodal NK/T cell lymphoma, nasal type, is an uncommon lymphoma with a broad morphologic spectrum and Epstein-Barr virus (EBV) can be demonstrated in the majority of tumors. NK/T cell lymphoma is most prevalent in Asia and Central and South America and affects males more commonly than females, with a median age of 50 years (range, $20-80$ years) [1-3]. NK/T cell lymphoma most commonly involves the nasal cavity and upper respiratory tract. However, this tumor can also arise in the skin, gastrointestinal tract, testes, lungs, salivary glands, pancreas, soft tissue, and bone marrow [4, 5]. Most commonly, the primary tumor originates in the centrofacial region and later disseminates to regional lymph nodes or distant organs [6]. Lung involvement by NK/T cell lymphoma, primary or secondary, is very rare and seldom reported in the literature [6-11]. The clinical course of this disease is usually highly aggressive with only a better outcome in patients with localized disease $[2,11]$.

Morphologically, the lymphoma infiltrates in a diffuse pattern and is usually angiocentric and angiodestructive with coagulative necrosis and apoptotic bodies. The cytological spectrum of the tumor cells is very broad, but monomorphic in individual cases, and consisting of either small-, medium-, large-sized, or anaplastic-appearing tumor cells. The cells in NK/T cell lymphoma are most commonly positive for $\mathrm{CD} 2$, cytoplasmic $\mathrm{CD} 3 \varepsilon$, and $\mathrm{CD} 56$, as well as cytotoxic granule-associated proteins such as granzyme B, TIA1, and perforin. However, tumors that are positive for $\mathrm{CD} 3 \varepsilon$, cytotoxic proteins, and $\mathrm{EBV}$, but negative for CD56, 
are also classified as NK/T cell lymphoma, nasal type, since they are clinically similar to CD56-positive cases. T cell receptor genes are not rearranged in the NK cell type, but are rearranged in the $\mathrm{T}$ cell type $[1,2]$.

Herein, we report a case of extranodal NK cell lymphoma, nasal type, presenting with massive secondary lung disease and bone marrow involvement. Although the patient was diagnosed with a retroperitoneal tumor 2.5 years prior to this diagnosis, he never had nasal and upper respiratory tract disease.

\section{Case report}

The patient was a 41-year-old Caucasian male who presented to a local hospital with dizziness and fainting spells. A chest X-ray showed pulmonary infiltrates and he was initially treated with antibiotics. However, his condition did not improve and he was referred to Zagreb University Hospital Center for further studies. At the time of admission, he was bedridden with fevers, thoracic and abdominal pain, and coughing foamy sputum with traces of blood. A computed tomography (CT) scan showed massive infiltrates in both lungs, measuring up to $8 \mathrm{~cm}$ in diameter, without significant mediastinal and peripheral lymphadenopathy, and careful examination of the nasal and upper respiratory tract was normal.

An open lung biopsy was performed and showed a diffuse, angiocentric, and angiodestructive infiltrate composed of large atypical lymphoid cells with round to slightly irregular nuclei, finely granular chromatin, small nucleoli, and moderate amounts of clear cytoplasm. Numerous apoptotic bodies and focal areas of coagulative necrosis were noted (Fig. 1). Immunohistochemically, the tumor cells were positive for $\mathrm{CD} 2, \mathrm{CD} 3 \varepsilon$ (Fig. 2), CD56 (Fig. 2, inset), TIA1, perforin, granzyme B, and MUM-1. The cells were negative for $\mathrm{CD} 5, \mathrm{CD} 7, \mathrm{CD} 4, \mathrm{CD} 8, \mathrm{CD} 20$, PAX-5, TdT, bcl-2, bcl-6, and CD10. EBER in situ hybridization for EBV was strongly and diffusely positive in the tumor cells (Fig. 1, inset), and polymerase chain reaction for $\mathrm{T}$ cell receptor gamma chain gene rearrangement showed a polyclonal pattern. Therefore, the patient was diagnosed with extranodal NK cell lymphoma, nasal type.

Examination of the bone marrow showed infiltration by tumor cells diffusely as single cells and in small clusters. Cytologically, the cells were medium-sized with round nuclei, moderately clumped chromatin, small nucleoli, and moderate amounts of pale cytoplasm containing fine azurophilic granules. The cells were positive by immunostaining for $\mathrm{CD} 2, \mathrm{CD} 3 \varepsilon$, and granzyme $\mathrm{B}$ and negative for CD5, CD7, and CD56. The peripheral blood showed mild pancytopenia without circulating tumor cells.
A CT scan of the abdomen and pelvis demonstrated a retroperitoneal tumor measuring $20 \mathrm{~cm}$ in diameter. The patient then admitted that he had been diagnosed with a $5-\mathrm{cm}$ retroperitoneal tumor 2.5 years prior, but had refused a biopsy and opted for alternative nutritional therapy. Since the clinical stage was IVB with bulky disease and he had high-risk disease according to the International Prognostic Index (IPI), he was started on CHOP-like chemotherapy consisting of dose-escalated cyclophosphamide and doxorubicin and standard doses of bleomycin and vincristine. In addition, intrathecal methotrexate was given for central nervous system prophylaxis. The patient improved initially, and the retroperitoneal tumor decreased in size, but then he developed a neutropenic fever and respiratory insufficiency. Despite intensive supportive measures and broadspectrum antibiotics, he died 10 days after the start of chemotherapy.

\section{Discussion}

This report describes a patient with extranodal NK/T cell lymphoma, nasal type, who presented with massive lung involvement, as well as bone marrow involvement, 2.5 years after the detection of a retroperitoneal mass. Even though the retroperitoneal mass was not biopsied at that time, it appears that the retroperitoneum was the primary site of the lymphoma. After an indolent clinical course, the patient developed massive lung involvement, rapidly deteriorated, and died shortly after beginning chemotherapy.

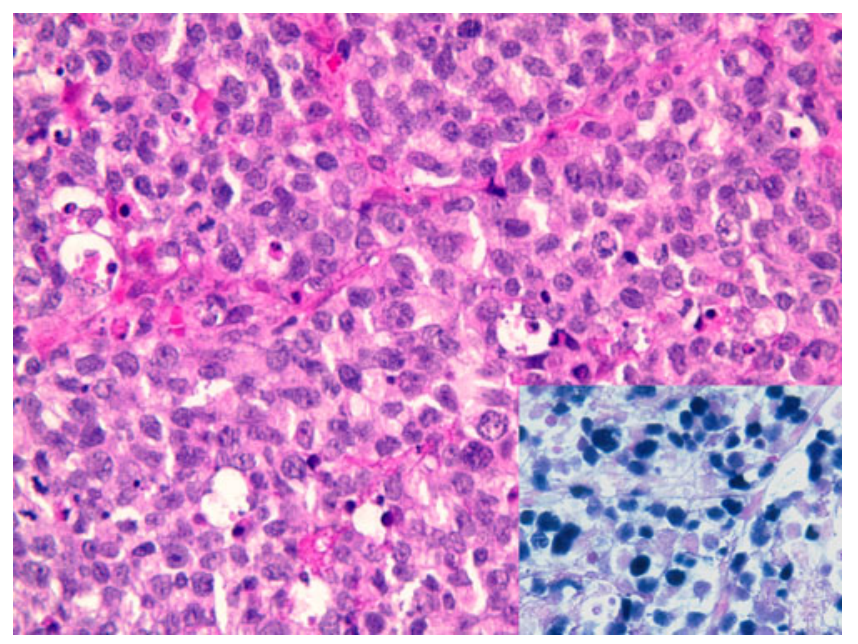

Fig. 1 NK cell lymphoma, nasal type, composed of medium-sized cells; numerous apoptotic bodies are present (hematoxylin-eosin stain, original magnification $\times 400$ ). Inset the EBER stain for EBV shows strong, diffuse nuclear positivity in the lymphoma cells (in situ hybridization, original magnification $\times 200$ ) 


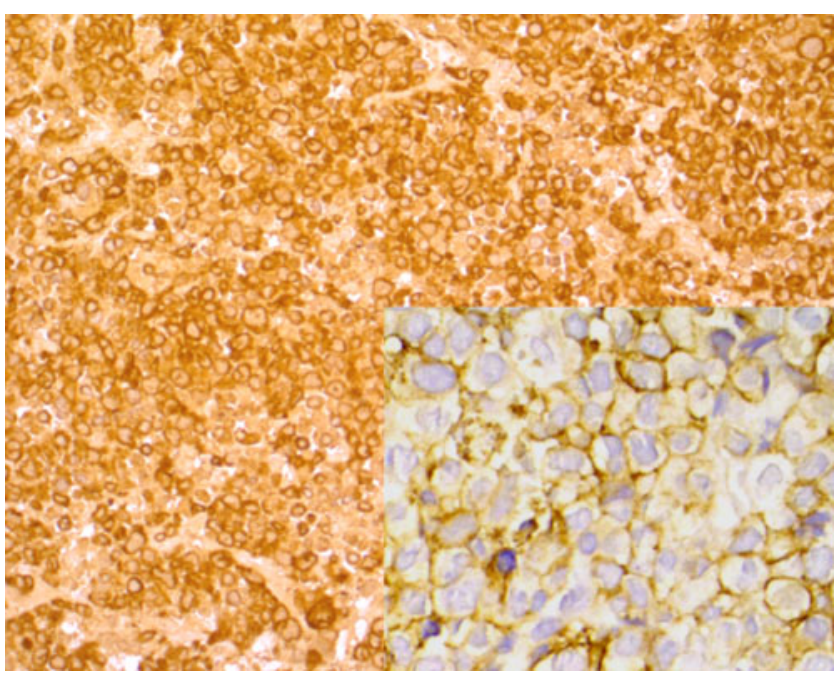

Fig. 2 A $\mathrm{CD} 3 \varepsilon$ immunostain shows strong, diffuse cytoplasmic positivity in the lymphoma cells (hematoxylin-eosin stain, original magnification $\times 200$ ). Inset CD56 immunostain shows membrane positivity in the lymphoma cells (hematoxylin-eosin stain, original magnification $\times 400$ )

Lee et al. [11] have recently proposed the classification of extranodal NK/T cell lymphomas into two categories: upper aerodigestive tract lymphoma (UNKTL) that includes the nasal cavity, nasopharynx, larynx, pharynx, and oral cavity; lymphomas occurring at all other sites were categorized as extra upper aerodigestive NK/T cell lymphomas (EUNKTL). The majority of patients $(85 \%)$ were categorized as UNKTL, whereas $15 \%$ had EUNKTL with involvement of various sites, such as the soft tissue and gastrointestinal tract, and four patients had a primary lung presentation. The EUNKTL group had more advanced stage disease at diagnosis, higher serum lactate dehydrogenase (LDH) levels, higher IPI scores, poorer performance, an inferior response to anthracycline-based chemotherapy, and shorter 5-year overall survival when compared to the UNKTL group. Although optimal treatment strategies for patients with extranodal NK/T cell lymphoma are not well defined, those in the EUNKTL group require aggressive therapy [12].

At the time of first presentation, our patient had a retroperitoneal tumor that measured $5 \mathrm{~cm}$ by CT scan, but which had enlarged to $20 \mathrm{~cm}$ at the time of the lung biopsy. Even though staging was not performed at that time, he presumably had localized retroperitoneal disease. His performance status at initial presentation was good, and he refused any medical treatment. According to the abovementioned classification, a primary retroperitoneal origin placed him into the unfavorable EUNKTL group. Even though a majority of these patients have an aggressive clinical course, there is a subset of patients who survive longer. Several factors seem to influence the survival in these patients, the most important being low clinical stage, good performance status, and a normal LDH level [4, 13, 14]. In clinical studies focusing on NK/T cell lymphoma originating in the upper aerodigestive tract, it has been shown that patients with low clinical stage (IE or IIE), good performance status, and no evidence of local tumor invasion have a better prognosis $[15,16]$. Another factor associated with better survival in patients with $\mathrm{NK} / \mathrm{T}$ cell lymphoma is an increased number of regulatory $\mathrm{T}$ cells in the tumor [17]. We postulate that the prolonged survival in our patient was related to the localized and relatively small tumor at the time of initial presentation. In addition, his good performance status put him into a more favorable survival category. Although the lack of a tumor biopsy at initial presentation creates some uncertainty in this case, the documented enlargement of the tumor from 5 to $20 \mathrm{~cm}$ is, in our opinion, evidence that the retroperitoneum was the primary site of the NK cell lymphoma. This is further supported by the decrease in size of the retroperitoneal tumor after the chemotherapy was started.

Regardless of the primary location, extranodal NK/T cell lymphoma presents as localized disease in approximately two thirds of patients $[6,12]$. Local extension of the tumor is more common than distant involvement and regional lymph node spread is a common occurrence [6, 18]. Dissemination of NK/T cell lymphoma is rare and appears to be organ specific, with the most commonly involved secondary sites being the skin, gastrointestinal tract, liver, bone marrow, and central nervous system [6, 9, 10, 18]. Lung involvement by $\mathrm{NK} / \mathrm{T}$ cell lymphoma is very rare and most commonly occurs with widely disseminated disease $[6,9,10]$. NK/T cell lymphoma arising in the lung is extremely rare and only a few cases have been reported in the literature $[7,8,11]$. Lung involvement, especially in disseminated disease, carries a poor prognosis for the patient $[6,9,10]$.

Aviles et al. [6] reported that patients with disseminated NK/T cell lymphoma may benefit from aggressive combination therapy. However, despite aggressive chemotherapy and intensive supportive measures, our patient died shortly after the beginning of therapy. The burden of tumor in his lungs and retroperitoneum was very large, and necrosis of the tumor due to chemotherapy caused severe respiratory insufficiency which led to his death.

In conclusion, when dealing with extranodal $\mathrm{NK} / \mathrm{T}$ cell lymphoma arising outside of the upper aerodigestive tract, one should pursue more aggressive initial chemotherapy, even in patients with localized disease. Novel agents and innovative therapies need to be developed for this aggressive lymphoma. Lung involvement by NK/T cell lymphoma is very rare, most commonly occurring in widely disseminated disease, and usually carries a poor prognosis for the patient. 


\section{References}

1. Chan JKC, Quintanilla-Martinez L, Ferry JA, Peh SC (2008) Extranodal NK/T-cell lymphoma, nasal type. In: Swerdlow SH, Campo E, Harris NL, Jaffe ES, Pileri SA, Stein H, Thiele J, Vardiman JW (eds) World Health Organization classification of tumors: tumors of haematopoietic and lymphoid tissues. IARC, Lyon, pp 285-288

2. Nava VE, Jaffe ES (2005) The pathology of NK-cell lymphomas and leukemias. Adv Anat Pathol 2:27-34

3. Pagano L, Gallamini A, Trape G et al (2006) NK/T-cell lymphomas nasal type: an Italian multicentric retrospective study. Ann Oncol 17:794-800

4. Al-Hakeem DA, Fedele S, Carlos R, Porter S (2007) Extranodal NK/T-cell lymphoma, nasal type. Oral Oncol 43:4-14

5. Suzuki R, Takeuchi K, Ohshima K, Nakamura S (2008) Extranodal NK/T-cell lymphoma: diagnosis and treatment cues. Hematol Oncol 26:66-72

6. Aviles A, Neri N, Fernandez R et al (2003) Nasal NK/T-cell lymphoma with disseminated diseases treated with aggressive combined therapy. Med Oncol 20:2013-2017

7. Laohaburanakit P, Hardin KA (2006) NK/T cell lymphoma of the lung: a case report and review of literature. Thorax 61:267-270

8. Lee BH, Kim SY, Kim MY et al (2006) CT of nasal-type T/NK cell lymphoma in the lung. J Thorac Imaging 21:37-39

9. Kuwabara H, Tsuji M, Yoshii Y et al (2003) Nasal-type NK/T cell lymphoma of the orbit with distant metastases. Hum Pathol 34:290-292

10. Se C, Lee SY, Choi JH et al (2002) Cutaneous dissemination of nasal NK/T-cell lymphoma with bone marrow, liver and lung involvement. Clin Exp Dermatol 27:120-122
11. Lee J, Suh C, Park YH et al (2006) Extranodal natural killer T-cell lymphoma, nasal type: a prognostic model from a retrospective multicenter study. J Clin Oncol 24:612-618

12. Lee J, Park YH, Kim WS et al (2005) Extranodal nasal type, NK/T-cell lymphoma: elucidating clinical prognostic factors for risk based stratification of therapy. Eur J Cancer 41:14021408

13. Au W, Weisenburger DD, Intragumtornchai T et al (2009) Clinical differences between nasal and extranasal natural killer/T-cell lymphoma: a study of 136 cases from the International Peripheral T-Cell Lymphoma Project. Blood 113:3931-3937

14. Kim TM, Lee SY, Jeon YK et al (2008) Clinical heterogeneity of extranodal NK/T-cell lymphoma, nasal type: a national survey of the Korean Cancer Study Group. Ann Oncol 19:1477-1484

15. Cheung MMC, Chan JK, Lau W, Ngan RKC, Foo WWL (2002) Early stage nasal NK/T-cell lymphoma: clinical outcome, prognostic factors, and the effect of treatment modality. Int J Radiat Oncol Biol Phys 54:182-190

16. Kim TM, Park YH, Lee SY et al (2005) Local tumor invasiveness is more predictive of survival than International Prognostic Index in stage IE/IIE extranodal NK/T-cell lymphoma, nasal type. Blood 106:3785-3790

17. Kim WY, Jeon YK, Kim TM et al (2009) Increased quantity of tumor-infiltrating FOXP3-positive regulatory $\mathrm{T}$ cells is an independent predictor for improved clinical outcome in extranodal NK/T-cell lymphoma. Ann Oncol 20:1688-1696

18. Barrionuevo C, Zaharia M, Martinez MT et al (2007) Extranodal NK/T-cell lymphoma, nasal type: study of clinicopathologic and prognosis factors in a series of 78 cases from Peru. Appl Immunohistochem Mol Morphol 15:38-44 